\title{
Contributing to a Tactile Language: Partners Communicative Accommodation to a Bodily/Tactile modality
}

\author{
Caroline Lindström
}

\begin{abstract}
This article is based on the master thesis "Contributing to a Bodily/Tactile Language by Transforming Cultural Customs: - A case study of Partners Communicative Accommodations in Socialised Praxises based on a Bodily/Tactile Modality and its Influences on a Bodily/Tactile Culture" (Lindström, 2017). The study is an ethnographic case study, based on a theoretical framework of language and culture. The case study involves a person with congenital deafblindness and ten of his care workers.

A cultural perspective was used as a way to describe the transformation of customs the care workers have to do to accommodate from their seeing/hearing perspective into a shared bodily/tactile modality. The transformation revealed different bodily/tactile strategies that the caregivers used together with the person with congenital deafblindness.

The case study was conducted in a longitudinal perspective using ten video clips within the same case study. The video clips were transcribed and analysed using an applied themebased analysis in the search for categories and themes that could help reveal the customs and accommodations.

The results of this study indicated several strategies that the care workers used together with the person with congenital deafblindness to transform customs in visual sign language into a bodily/tactile communication. The strategies consisted of different body alignments, (hand-over-hand) positions, turn taking markers, the directing of hand and bodies in the conversation, touch and positions from the peripheral partner, the use of objects in the conversation, Martin's body as an articulation place, haptic signals, back and forth articulation, different tempo and rhythm as well as repetitions.
\end{abstract}

\section{Keywords}

Congenital deafblindness, language development, tactile language, tactile sense, cultural customs 


\section{Introduction}

This study sheds light over the communication between a person with congenital deafblindness (cdb) and his care workers. In this study the care workers are known as communication partners to highlight more equal roles in the communication and development of language. The encounter between a person with congenital deafblindness and a communication partner is an encounter between two people using different sensory modalities. The differences between them can create communication difficulties which can be hard to overcome without the right support and interventions. Because of the importance of right support this ethnographic longitudinal case study is focused on congenital deafblindness and language development and how the partner is contributing to the development.

Deafblindness is a combined vision and hearing impairment, which is defined as a distinct disability. It affects social life and communication and therefore requires special services and competence from the society to grant access to activities and participation. (Nordic welfare center, 2017). The combined vision and hearing impairment makes it difficult for persons with deafblindness to use the impaired senses to compensate one with the other. This means that the use of their tactile sense becomes important in various degrees to help compensate the combined impairment. In congenital deafblindness the impairment in both senses is present before a language has been acquired. Therefore, people with cdb cannot rely on their residual visual and/or auditory senses for developing communication and language. Instead, the bodily/tactile sense becomes their primary modality for experiencing and understanding the world and their preferred communication mode is, therefore, a bodily/tactile language.

Since the middle of the 90's, the research field within cdb, has been investigating and demonstrating the expressions that emerge from people with cdb (Nafstad \& Daelman, 2017), and continually been focusing on bodily movement and gestures from persons with cdb in a language development perspective (Dammeyer, Nielsen, Strøm, Hendar \& Eiríksdóttir, 2015; Schjöll Brede \& Souriau, 2016). However, in the light of both persons equal contribution, we also need additional knowledge of how to describe the ways the communication partner exposes a cultural language to the individual with congenital deafblindness in a bodily/tactile modality (Ask Larsen, 2015).

\section{Background}

The main reason for this study is grounded in a desire to unfold more knowledge about how the partners are contributing to the co-creation of a bodily/tactile language. The view of this process is that tactile language is developed in interaction with people with congenital deafblindness. 


\section{Language development from a dialogical perspective}

This study was conducted from a dialogical perspective of language development. The dialogical perspective is emphasizing both partner's recognition of the other and willingness to move closer to the other person's perspective of the world. In language development between a person with $\mathrm{cdb}$ and a seeing/hearing partner, the different perspectives of the persons will be based on different sense modalities when experiencing the world. Hart (2010) describes this process of co-creation of a bodily/tactile language when he states that it consists of an equal contribution from both partners, where the person with deafblindness brings tactile and bodily movements and gestures, based on their perception of the world (Forsgren, Daelman \& Hart 2018) and the non-deafblind partner brings elements from an existing cultural language, as illustrated in figure 1 . This means to overcome the mismatch in perspectives of the world it is incumbent on the communication partner to move into the tactile world since the person with cdb cannot journey to the communication partner's perceptual perspective of the world (by vision and hearing) (Hart \& Rodbroe, 2010). This concludes that a modality based on touch is unavoidable for meaning making and for developing a tactile language together with a person with $\mathrm{cdb}$. It is therefore logical that the partner must use tactile adaptations of visual signs in order to contribute with elements from an existing cultural language in a bodily/tactile modality to make the language input understandable and meaningful for the person with cdb.

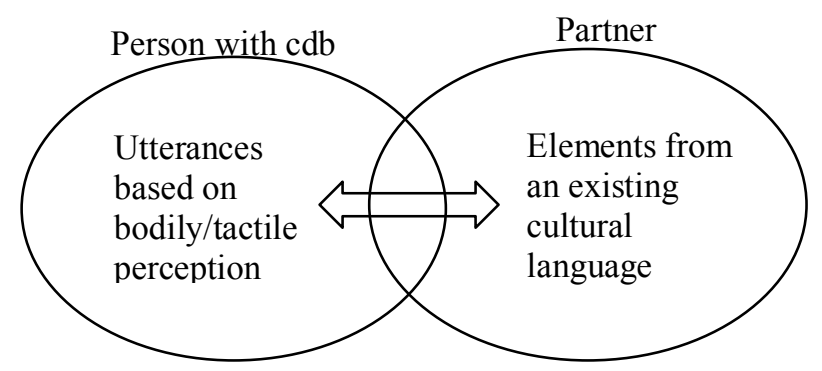

Figure 1. Illustration of both partner's contributions to a co-creation of a tactile language in a dialogical perspective.

\section{Cultural impact on language acquisition}

The process of moving into the tactile world means to transform your known customs from a hearing/seeing modality into a tactile modality by tactile adaptations of visual sign language. To explain and illustrate the transformation process from one modality to another a research field called Language Socialization has been included in the theoretical framework of this study. The researchers and founders of the field, Schieffelin and Ochs, wanted to illustrate that there is diversity in cultures regarding customs when socializing with children and that the customs thereby are affecting children's language acquisition (Ochs \& Schieffelin, 1983; Ochs \& Schieffelin, 1984; Ochs, 1988). In general, this is not an issue since the children and adults share the same language and culture as the social 
environment and their previous generations. Though, it is an issue for congenitally deafblind people and their partners where the mapping between language and culture has to be built up from scratch (Souriau, Rodbroe \& Janssen, 2010). In the aim of capturing the social and cultural structures Ochs et. al. (2005) are presenting different dimensions within child directed communication (CDC). Two of the dimensions included in CDC are participation framework and semiotic repertoires, which have been chosen for the analytic focus of this study.

Participation framework. The participation framework includes both specific roles in the interaction and different corporeal alignments involving children and their caregiver. The roles in the interaction refer to communicative and activity-specific roles that children and other participants assume in the interaction (Ochs et al., 2005). In interaction between a person with cdb and a partner Nafstad and Rodbroe (2013) are emphasizing the importance of the different roles as "the speaker", the "listener" and "the thinker". The authors underline the importance of knowing the differences between the roles and how to give a person with cdb access to all different roles in tactile/bodily interaction.

Corporeal alignments mean that people use different positions and orientations in interaction (see figure 2) and these positions are culturally created and different throughout the world.
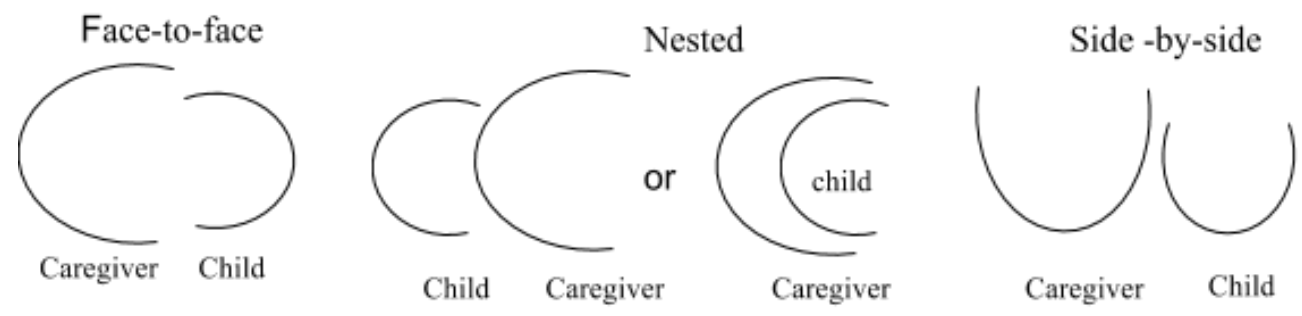

Source: Ochs et al. (2005) This illustration over the participation framework within CDC is demonstrating different corporeal alignments that caregivers and children can have in the interaction.

Figure 2. Several types of socio-culturally organized corporeal arrangements involving children.

Interaction and communication with persons with $\mathrm{cdb}$ has to be touch based. Gregersen (2018) call this touch based interactions 'Body-with-body interactions'. Tactile conversations include the whole body and hands which means that both persons have to be oriented towards each other by physical contact to speak and listen in the dialogue. A conversational practice in a bodily/tactile modality is therefore dependent on how the communication partners are related towards each other and in what way they are in touch. Different terms are used to describe and explain a tactile conversation, for example four-handed communication (Mesch, 1998, Raanes, 2006); hand-under-hand (Miles, 2003) or hand-over hand (Nafstad \& Rodbroe, 2013). In this study the hand-over -hand term is being used to describe the hand positions of Martin and the partner in their conversation. 
Semiotic repertoires. A semiotic tradition studies communication from a broad perspective including both linguistic and non-linguistic systems. Based on different communication modalities (visual, auditory, gestural) people have access to various semiotic repertoires, which includes many forms such as pointing, eye gaze, facial expression, rhythmic movements and vocalization. In the interaction with people with $\mathrm{cdb} a$ bodily/tactile modality is used for communication including a bodily/tactile repertoire for example pressure, speed, rhythm, gestures, bodily expressions and tactile signs.

It has been stated that from a dialogical perspective on language development together with a person with $\mathrm{cdb}$, the partner has to transform signs from visual sign language into a tactile/bodily modality so it can be perceived by the person with cdb. The structure of a sign in visual sign language consist of three simultaneous aspects: articulator, articulation place and articulation. Articulator means different handshapes, articulation place is the place where the sign is performed and the articulation is movements of the hand(s) when performing the sign (Stokoe, 2005).

\section{Method}

\section{Design}

For this study influences from the regular method for research in ethnography, longitudinal fieldwork, (Hill \& Millar, 2015) was combined with a commonly used method within the deafblind field, case study (Rodbroe \& Janssen, 2008). The combination was performed through a case study using different video clips within a longer period including different ages of the person with cdb. That way the case was seen from a longitudinal perspective and the term ethnographic case study was therefore adopted as a way of describing the design of this study (Hill \& Millar, 2015)

Participants. The case in this study is about a young man born in 1999, and ten of his communication partners. A fictitious name, Martin, has been given to the young man in this study in respect of confidentiality. For the same reason the communication partners who participate in the video clips also have fictitious names (see table 1). Martin has a combined severe visual and hearing impairment and he also has a condition that affects his muscles for which the use of a wheelchair is now necessary. Martin's predominant source for communication is by a bodily/tactile modality. He uses tactile signs and expressions, body language, gestures, own expressions, sounds, mimic and actions to communicate with his partners. His communication partners have used, and are using, a combination of tactile sign language, Martin's own expressions, tactile symbols and sounds when communicating with him. However, the main focus in the communication is touch based (Lindström, 2017).

Procedure and data collection. In this study a collaboration with other persons regarding transcription, analysing the data and finding categories and themes, has been used to secure validity through an independent assessment of the data. Two persons in the latest 
film clips (see Table 1, video 7-9) have been asked to read through the transcriptions of those films for the purpose of a second opinion of the transcribed data and to get their consent with the interpretation of the film clip. Furthermore, a co-researcher has been involved in the process of analysing the data and finding categories and themes.

Both old and new film clips have been used as data in this study. The older film clips of Martins as a young boy were collected by contacting a person within the field who has been involved in his life. No particular criteria for selection were applied to the older film clips as the amount were limited. However, the older film clips have been used as good examples in the past stating that they had a good quality in relation to communication focus. The new film clips were recorded where Martin attend school nowadays and the film clips were selected based on Martin's interest and willingness to communicate tactilely over a longer sequence. When basing the selection on Martin's interest and focus it is assumed the the partners are interacting in an exemplary way. The selection was also made with the aim to use both oneto - one situations and multi-party conversations. An overview over the selected film clips can be seen in table 1 .

\section{Table 1}

Overview of the selected film clips

\begin{tabular}{|c|c|c|c|c|}
\hline Letter & Video & Time & Year (Martin's age) & Participants \\
\hline A & $\begin{array}{l}\text { Where is } \\
\text { Lina? }\end{array}$ & $00: 40$ & $2003(4)$ & Martin, Julia \\
\hline B & $\begin{array}{l}\text { Take the } \\
\text { shoes }\end{array}$ & $2: 10$ & $2005(6)$ & Martin, Lina, Marie \\
\hline $\mathrm{C}$ & $\begin{array}{l}\text { Wheel on the } \\
\text { bus }\end{array}$ & 03:13 & $2006(7)$ & Martin, Anna, Marie \\
\hline D & $\begin{array}{l}\text { Martin has } \\
\text { baked a cake }\end{array}$ & $00: 27$ & $2008(9)$ & Martin, Petra, Sandra \\
\hline $\mathrm{E}$ & $\begin{array}{l}\text { Singing } \\
\text { together }\end{array}$ & $03: 32$ & $2012(13)$ & Martin, Linda, Fredrik \\
\hline $\mathrm{F}$ & $\begin{array}{l}\text { Lighting } \\
\text { candles }\end{array}$ & 02:07 & $2012(13)$ & Martin, Linda, Fredrik \\
\hline G & Massage oil & $02: 42$ & 2017 (18) & Martin, Tove \\
\hline $\mathrm{H}$ & Hot cocoa & $02: 29$ & 2017 (18) & Martin, Tove, Ellen \\
\hline I & Bird food & 00:49 & 2017 (18) & Martin, Tove, Ellen \\
\hline
\end{tabular}

\section{Data Analysis}

An exploratory approach (Guest, 2012) was chosen for sorting and analysing the data and was adopted from Raanes (2006) doctoral study where she describes a process for notation 
and transcription of data in three different steps: Step 1 is an overview over the content and includes a manual notation where the researcher goes through the material and marks sequences of special interest. In this first step observable findings in the data regarding participation framework and partners' adjustments in the semiotic register were marked to gain a first overview of the data. Step 2 is an observation of the communication situation, where the communication situation is related to both the situation and outer factors. This step was necessary for the analysis when describing the participation framework and was described in the introduction to every transcribed film sequences. Step 3 involves the transcription of the bodily/tactile communication.

Transcription. Raanes (2006) concludes difficulties in transcribing tactile sign language due to differences in transforming speech and visual sign language into text compared to tactile sign language. The difficulties are related to no existing conventional transcription system developed for tactile sign language and difficulties of capturing all the details in tactile conversation by a visual medium. As a result of the obstacles there have been different approaches to a transcription of tactile sign language where researchers have developed and tried different ways of transforming bodily/tactile expressions into text and pictures to make it possible to describe the interaction at a micro level. (Ask Larsen, 2003: Brede, 2008; Mesch 1998; Raanes; 2006). For the transcription of the data in this study a combination of elements from a notations system in Conversation analysis (Norrby, 2014), suggestions for transcription system of tactile language (Mesch, 2003) and suggested principles for bodily/tactile language (Ask Larsen, 2003) have been used.

observable techniques related to cultural customs. When searching for categories and themes in data there are several approaches and techniques that can be used. To identify themes in transcription Ryan and Bernard (2003) are suggesting different observational techniques, which some have been adopted for the analysis of the data: Repetitions, Indigenous categories/typologies, Similarities and differences and Linguistic connectors. However, since the focus of this study concerns customs and manners in bodily/tactile interaction and conversations the author has adjusted the suggested techniques to better suit a cultural and linguistic approach with focus on semiotic register and corporeal alignments. The different approaches are explained in the examples below:

- Repetitions. Observation of repetitions of the same semiotic repertoires in a bodily/tactile modality in different or the same situation.

- Cultural domains. Observation of different ways of conversing bodily/tactilely. The researcher looks for customs within corporeal alignments and/or semiotic repertoires in the interaction that are used in familiar ways (within the deafblind field) or creative ways (in comparison with both visual sign language/speech and tactile communication). 
- Relationships. Observation of different relationships in the interaction affecting the customs used in a bodily/tactile modality. Observations of functions in the semiotic repertoires or corporeal alignments (Ex. $\mathrm{x}$ is a means of affecting y), spatial orientations within the corporeal alignments (Ex. $\mathrm{x}$ is near y) and operational definitions of the corporeal alignments and/or semiotic repertoires (Ex. $\mathrm{x}$ is a tool for doing y).

- Similarities and differences. The researcher compares the repetitions, cultural domains, and relationships, both in different parts of the same video, but also between the different video recordings (Lindström, 2017).

Four different themes with associated categories were identified from the data using the above described techniques. The themes and subcategories are presented in table 2.

\section{Table 2}

Identified categories and themes

\begin{tabular}{|c|c|c|c|c|}
\hline Themes & $\begin{array}{l}\text { Tactile } \\
\text { conversational } \\
\text { positions }\end{array}$ & $\begin{array}{l}\text { Signing space and } \\
\text { references }\end{array}$ & Articulation places & Articulation \\
\hline \multirow[t]{4}{*}{ Subcategories } & $\begin{array}{l}\text { Body } \\
\text { alignments }\end{array}$ & $\begin{array}{l}\text { Turn taking } \\
\text { markers }\end{array}$ & $\begin{array}{l}\text { Martin's body as } \\
\text { an articulation } \\
\text { place }\end{array}$ & Back and forth \\
\hline & $\begin{array}{l}\text { Hand-over- } \\
\text { hand }\end{array}$ & $\begin{array}{l}\text { Directing hands } \\
\text { and bodies in the } \\
\text { conversation }\end{array}$ & $\begin{array}{l}\text { Articulation place } \\
\text { for haptic signals }\end{array}$ & $\begin{array}{l}\text { Tempo } \\
\text { Repetitions }\end{array}$ \\
\hline & & $\begin{array}{l}\text { Touch and } \\
\text { positions from the } \\
\text { peripheral } \\
\text { partner }\end{array}$ & & Rhythm \\
\hline & & $\begin{array}{l}\text { Using objects in } \\
\text { the conversation }\end{array}$ & & \\
\hline
\end{tabular}




\section{Results}

\section{Tactile conversational positions}

The results from the study are presented below in text and with illustrations. Additionally, there are photos illustrating signs in visual Swedish sign language which all come from the lexicon at Stockholm's university homepage (http://teckensprakslexikon.su.se).

Body alignments. Body alignments mean that people use different bodily orientations and positions when interacting. Figure 3 presents an overview over different body alignments uncovered in the data and the different body alignments are explained further in the text below.
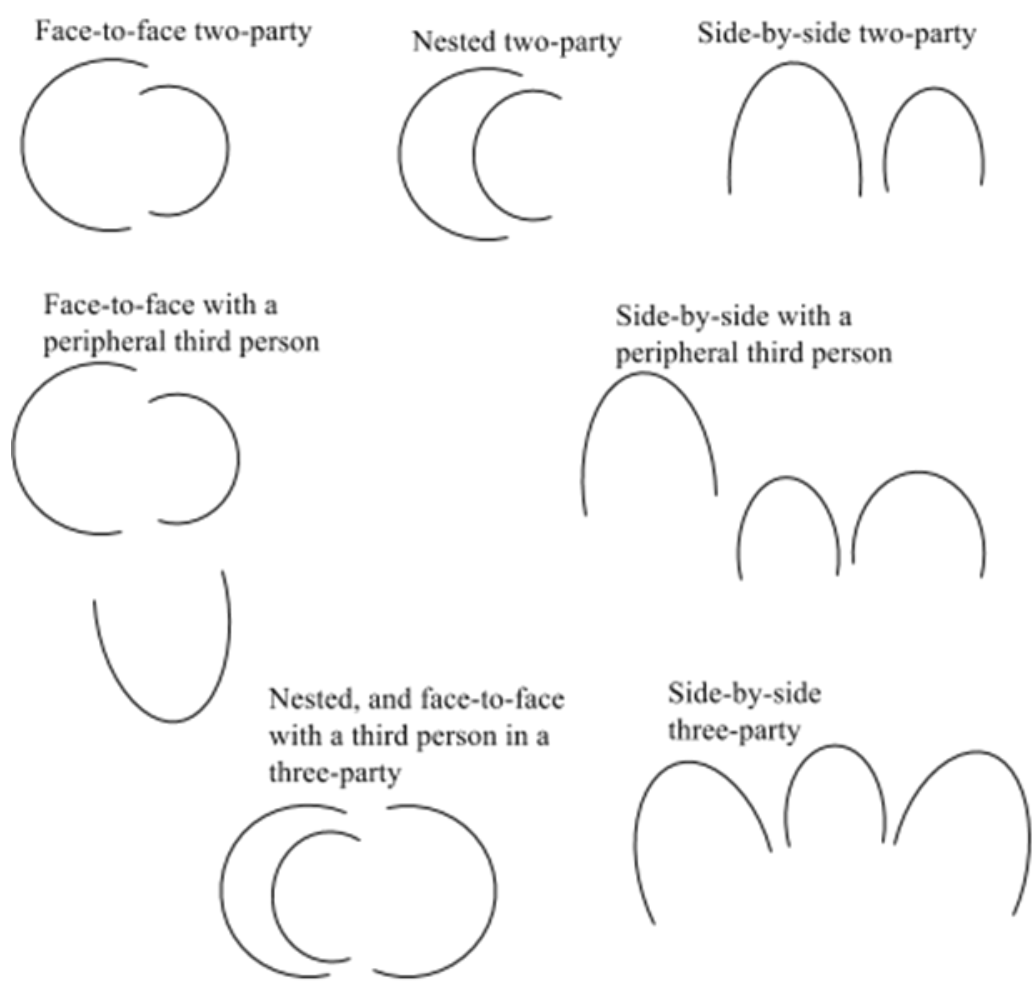

Source: Illustration made by the author based on Ochs et. al (2005) demonstration over different corporeal alignments that children and adults can have in their interaction

Figure 3. Illustration over identified body alignments in the data.

One of the identified body alignments, as seen in figure 3, was a face-to-face interaction between Martin and his partner where they were sitting near each other face-to-face touching each other's legs and hands. The face-to-face position was also identified when there was a peripheral third person. This means that a third person was present sitting near at the same time as the face-to-face interaction, but was not in a sustained physical contact with them. A face-to-face interaction is common also in tactile interaction with people with acquired deafblindness (Mesch, 2001; Raanes, 2006). The third person initiated physical contact with 
Martin occasionally but the main contact was between Martin and the partner in the face-toface interaction.

The nested position means that Martin was sitting on the partner's lap. One film also showed a mix between face-to-face alignment and a nested position, where Martin sits on one partner's lap and a second partner is sitting in front of them hence they are facing each other. The nested position could support stabilization for the child and enhances the partner's focus on the child's movements or vibrations in the chest. Aside from the nested position, there was also an example of a side-by-side alignment of Martin and his partner lying next to each other on the floor. In addition to the side-by-side alignment, there were also examples of a side-byside position with a third peripheral person. The persons in the different film clips were only sporadically in physical contact with Martin, similar to the peripheral person in the face-toface interaction. However, the peripheral person in the side-by-side alignment acknowledged her presence in other ways, for example, stamping the floor near Martin. Furthermore, there were examples in the video clips also identified as a three-party interaction where Martin and two partners were interacting with each other in a side-by-side position with Martin sitting in the middle. Additionally, all described alignments include positions where Martin and his partners are either sitting or lying down.

Hand-over-hand. The hand over hand category was the most common category in the data. The frequency of the hand-over-hand is indicating a development of Martin's ability to participate in a tactile conversation practice. Throughout the whole transcribed material there were a lot of examples of how the partners are using different hand positions together with Martin. In many of the examples, they are using the hand-over-hand positions conversing tactilely. However, in the films from his early childhood (film A-C) the hand-over-hand position has not yet been established between them in a conversation and the partner is therefore using other positions as tactile strategies in their interaction, offering him opportunities to feel their hands in a listening and talking position on the basis of his present development in a tactile conversation modality. Illustration 1 below illustrates how the partner is inviting Martin to listen tactilely by placing her hand in contact with Martin's listening hand when performing the sign (Lindström, 2017). 

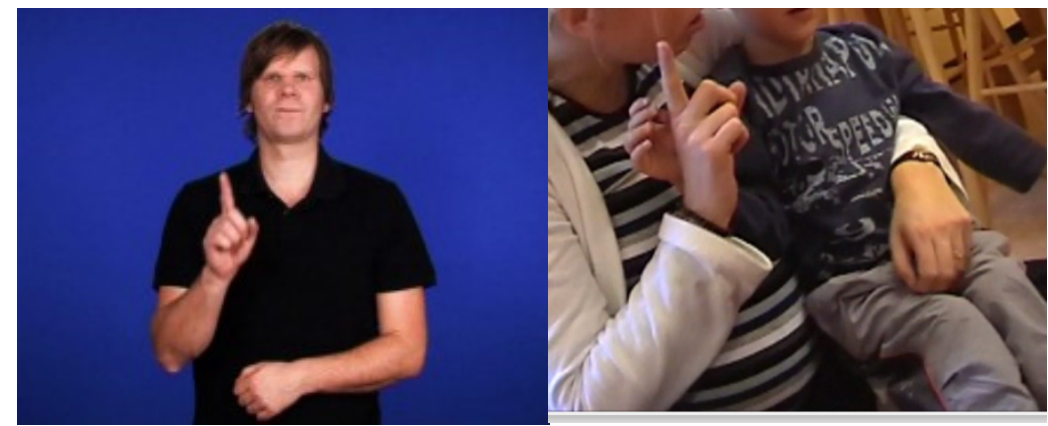

Source: Video A

Illustration 1. Example of a hand over hand position.

In addition to inviting Martin to listen tactilely, there were also examples in the earlier videos where the partner placed her hand on top of Martin's giving him opportunities to be in a talking position before a hand-over-hand position is established. The partner was also adjusting their hand positions when they were in contact tactilely to facilitate Martin's ability to hold on to the partner's bigger hand, for example by adjusting their hand position so that Martin holds around some of her fingers making it possible for him to follow her in their conversation. There was also an example where the partner signs with Martin when holding her hand in a listening position on top of his hand. This strategy is facilitating Martin's ability to perceive the sign despite that the hand-over-hand position is not established.

In the film material, when the hand-over-hand position was established, there were a lot of examples when the partner transforms visual signs into a tactile modality using hand-overhand with both hands. In addition to the established hand-over-hand position, there were also examples where the partner and Martin are using only one hand in their tactile hand-overhand conversation (see illustration 2). Illustration 2 firstly illustrates the Swedish visual sign for "bird food". In the photos Martin and his partner is standing near a branch where they have hung bird food. Martin is holding his left hand on the right hand of the partner as she tactilely signs BIRD-FOOD. 


\section{Swedish visual sign BIRD-FOOD}

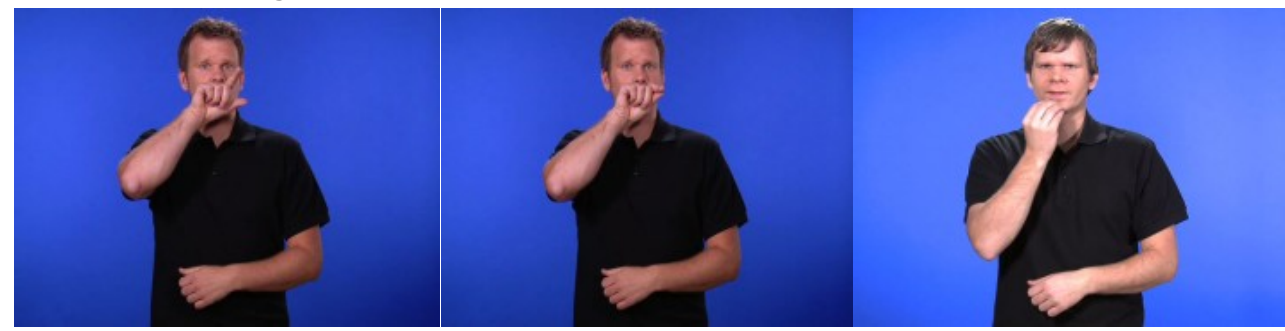

Partner signing BIRD-FOOD to Martin using one hand in the hand-over-hand position
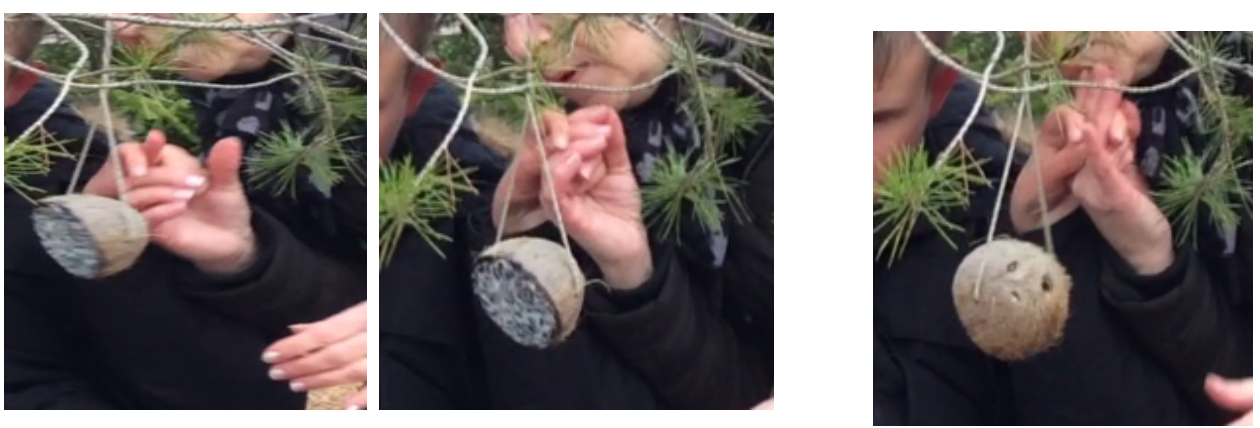

Source: Video I

Illustration 2. Example of a one handed hand-over-hand position.

In the videos when the hand positions with one hand or two hands have been established, Martin and his partners are alternating the different hand positions during the same film. The position using one hand could sometimes be explained by physical restrictions since the wheelchair is limiting the ability of positions and being near or face-to-face. In some situations, one hand was also used in the conversation simultaneously as acts or exploration by Martin. That way the partner is able to comment in the situation making the signs more relatable in the direct situation to what Martin perceives.

There were also examples of a more complex hand-over-hand position called a dialogue position, where both the participants in the tactile conversation have one hand in a talking position and one hand in a listening position (see illustration 3). In illustration 3 the partner is first talking holding her hands beneath Martin’ $s$, and Martin is listening holding his hands on top of the partner' $s$ hands. Martin then switches his right listening hand to a talking position by switching his hand from on top of the hand of the partner to beneath the hand of the partner. 

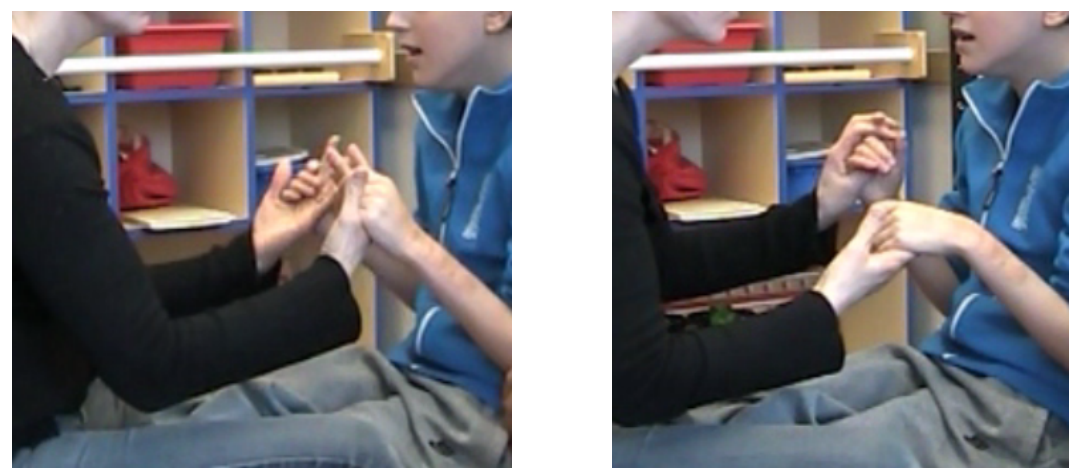

Source: Video E

Illustration 3. Example of a hand-over-hand position that changes to a dialogue position.

Lastly, a hand-over-hand position was identified in a multi-party conversation including three persons conversing tactilely at the same time (see illustration 4). In illustration 4 the two partners are conversing using hand-over-hand. The partner to the left is talking and the partner to the right is listening. Martin holds his hands and arms in a resting position on top of their hand and arms making it possible for him to overhear their conversation feeling their movements.

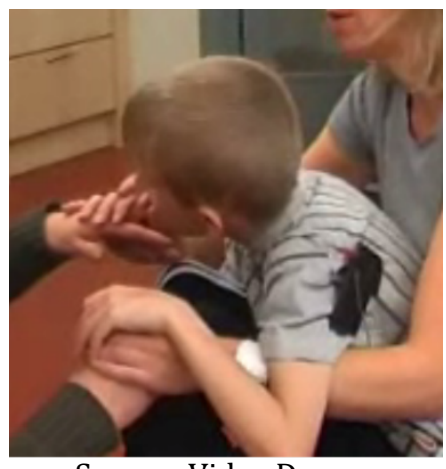

Source: Video D

Illustration 4. Example of a multiparty conversation in a tactile modality.

The presented use of different hand positions could be seen as a result of an increased complexity in their development of a tactile communication practice, since one hand and a dialogue position is more complex than using two hands (Mesch. 2011).

\section{Signing space and references}

This theme concerns the different tactile strategies the partner is using within the signing space. Some of the customs that were identified are connected to turn taking and the transformation from visual sign language to tactile communication. Another subcategory is directing hands and bodies in the conversation for the same function as pointing. Finally, the theme is also addressing how the third partner is using touch and positions in the signing 
space, as well as objects. The different themes are described in the text below with accompanying pictures.

Turn taking markers. Turn taking markers were not very common but still observed as important ingredients in their interaction. The partner is adjusting the hand positions in the signing space in different manners to mark for example pauses in the conversation or lifting the hands as an initiative for taking turn. However, the film material also revealed tactile strategies to mark turn taking positions before the hand-over-hand position was established, for example the partner stops after the song has ended, lower their hands and hold them still as a marker for that the turn has ended. Besides the identified tactile turn taking markers, there were also examples in the video material where the partner tried to take their turn as a speaker in the interaction by placing their hand beneath Martin's hand.

Directing hand and bodies in the conversation. In Swedish visual sign language pointing with the index finger is used to refer to things. This way of referring was also present when interacting with Martin in a tactile hand-over-hand position. There were also several examples where the partner is directing their hands and bodies in different ways in the conversation to refer to things and persons within the signing space. These hand and body movements are therefore having the same function in their tactile conversation as pointing to refer to objects or persons. For example, the partner was directing their hands to refer to the peripheral partner using touch as a confirmation of where that partner is in relation to Martin and the partner he is conversing with at that moment. In illustration 5 Martin, wearing a blue sweater, is sitting face-to-face with Linda. Fredrik sits at Martin's right side in the picture. Linda is directing Martin's hand so he can feel where Fredrik sits.

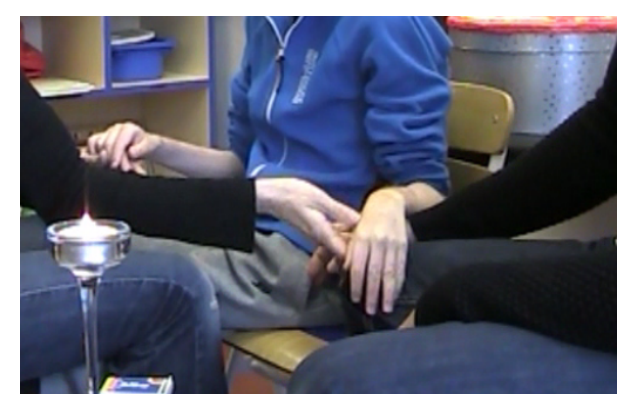

Source: Video F

Illustration 5. Example of referring to another person in a tactile modality.

Touch and positions from the peripheral partner. In the video material, there were several examples of the peripheral partner using touch or positions when being near Martin to enhance his chances to perceive that they are there. The partners were either using touch placing their hands gently on different parts of Martin's body or positioning themselves making their bodies near and/or in contact with Martin, acknowledging themselves and 
making themselves available for interaction and communication with him. In auditory or visual communication the children see or hear other people communicating all the time at distance. It is therefore important for the partners to be close enough to be able to acknowledge their presence to Martin in a bodily/tactile modality to give him access to social situations and communication in a wider scale.

Using objects in the conversation. Throughout the video material, the partners are also using objects in different ways when communicating and interacting with Martin. In some of the examples, the partner brought the objects closer to Martin so he could feel and explore them. In other examples, the partner used the objects in different ways in the situation making it a part of their playful interaction and communication.

\section{Articulation places}

This theme is describing different customs and strategies concerning how the partner is using articulation places when conversing tactilely with Martin. The theme is divided into the categories Martin's body as an articulation place and Articulation place for haptic signals which both are presented and described below with pictures and transcriptions from the videos.

Martin's body as an articulation place. When signing visually a sign has a certain articulation place where the sign is produced. It can be in the facial area, at the upper body, arms, hands or in the neutral field in front of the person. In the different videos, there were many examples where the partner transforms a visual sign using hand-over-hand placing the sign on her body or in the neutral field between them, as she would do if she signed visual. This was present both with one hand and two hands in the hand-over-hand position (see illustration 6).

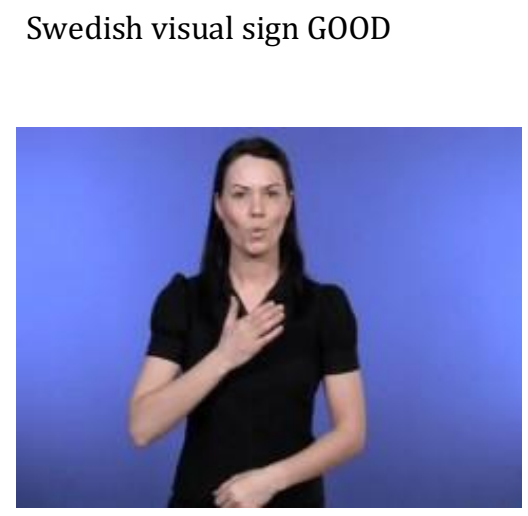

Source: Video E
Partner signing GOOD to Martin with two hands in hand-over-hand position

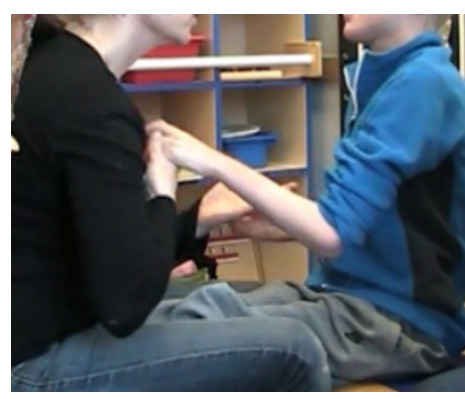

Illustration 6. Example of the sign GOOD in a tactile modality.

However, there were also examples where the partner used the conventionally articulation place as in the visual sign but placed the sign on Martin's own body when performing the sign, as in illustration 7. 
Swedish visual sign SWEATER

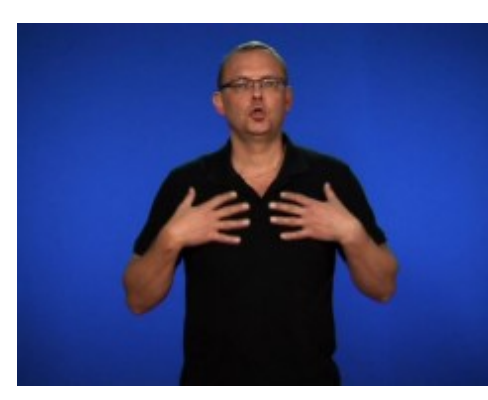

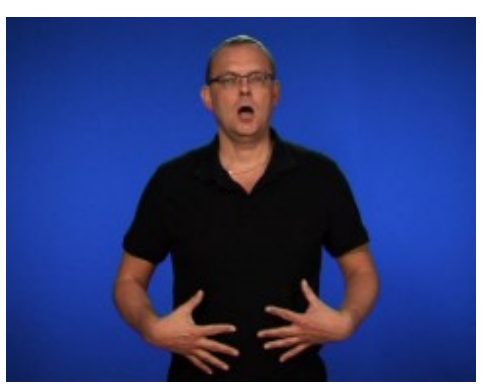

Source:Video F
Partner signing SWEATER to Martin with his chest as an articulation place

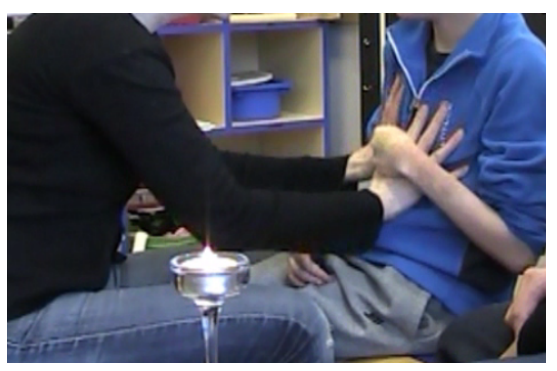

Illustration 7. Example of Martin's chest as an articulation place.

One example also revealed a strategy involving both the partner's body and Martin's body as articulation places at the same time in combination with a hand-over-hand dialogue position. The partners also used more creative ways of using Martin's body as an articulation place. In illustration 8 the visual signs TAKE-SHOE is performed in the neutral space in front of the signer in visual sign language. In the conversation with Martin, the partner instead placed the signs in contact with and on top of Martin's leg. 
Swedish visual sign TAKE-SHOE with the neutral field as the articulation place TAKE

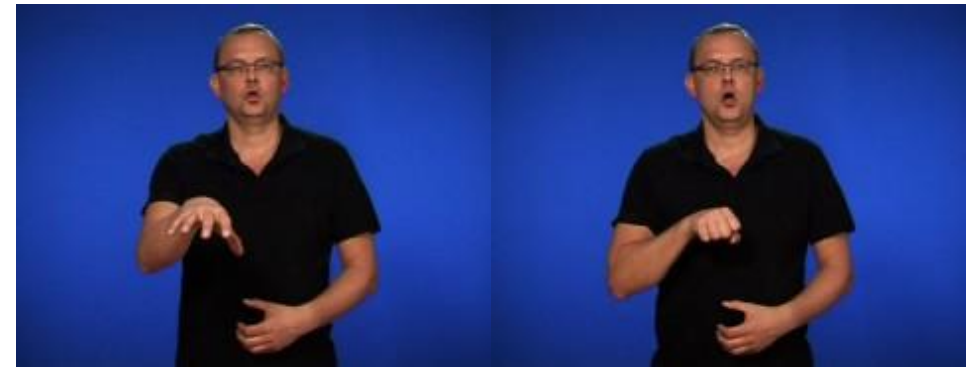

SHOE

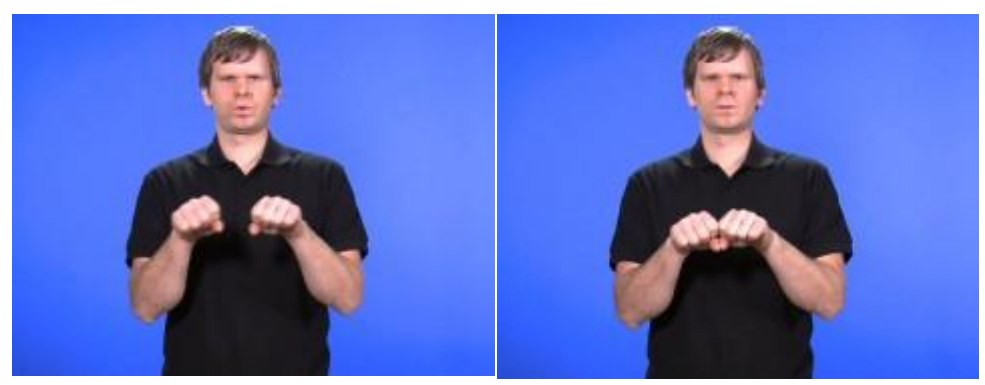

Partner signing TAKE-SHOE with Martin's leg as an articulation place TAKE SHOE
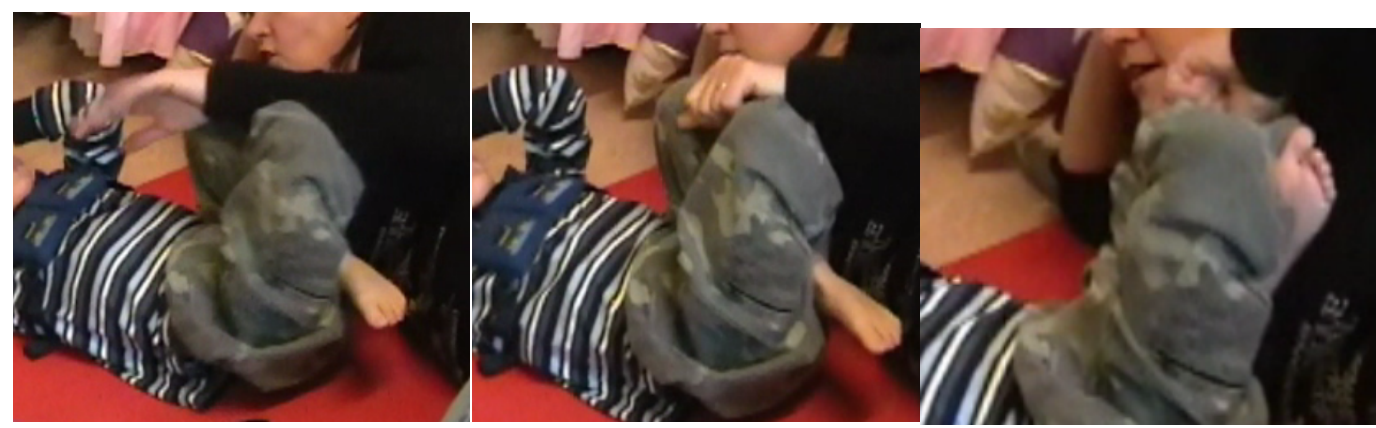

Source: Video B

Illustration 8. Example of Martin's leg as an articulation place.

Articulation place for haptic signals. In this study, the term haptic signals were used to describe tactile gestures that the partner use to inform Martin of things. Haptic signals usually consist of touch, movement, and pressure depending on the meaning of the signal. In the material, there was one example where the partner was performing this signal onto Martin's body. In the example the partner stands behind Martin who sits in a wheelchair communicating with another partner beside him. The partner behind him wants to back the wheelchair and to inform Martin of this she places her hand on his arm and presses back on his arm with a firm motion. 


\section{Articulation}

This theme concerns the different movements the partner is using in the bodily/tactile interaction with Martin and the variations of the movements. In visual sign language, a sign consists of a hand shape, a location (articulation place) and a movement (articulation). This theme is describing how the partner used different articulations together with Martin that was not just regular movements included in a tactile sign but used for different purposes in a bodily/tactile modality.

Back and forth. The category "back and forth" involves an articulation which means that the hand or the body moves in small or bigger movements from side to side or in a "wiggling motion". In the videos, there were examples of when the back and forth articulation were used to accentuate an expression, both with the whole body or with a hand performing a tactile sign onto Martin's body. The back and forth articulation was also identified as a tactile strategy to express and communicate about emotions. The wiggling motion of the hands or the whole body in the interaction was used in a playful, narrative manner to share positive emotions. In visual sign language, an accentuation is often expressed by facial expressions which indicate the necessity of an accommodation into bodily/tactile modality to compensate for features that otherwise would be lost.

Tempo. The category 'tempo' describes the speed by which the articulation of the gestures, expressions or signs were produced in the material. The different variations of the tempos included in the transcription were a quick motion, a slow motion and a fast and distinct motion. The different tempos of the articulation could be determined depending on the context of the whole communication situation. A slower pace was used in a calm situation for example talking about a massage or when the partner wanted to be extra clear in the performance of the sign. A faster pace was shown in those cases where the context where more playful or to further accentuate an expression in combination with the back and forth movement in a distinct motion.

The use of different tempo and variations of speed is closely linked to narrativity which has an important role in development for all children including children with cdb (HanningZwanenburg, Rodbroe, Nafstad and Souriau, 2016).

Repetitions. The category 'repetitions' is included in the theme articulations because the motion in a sign or a gesture is easier to perceive in a bodily/tactile modality compared to a handshape or the articulation place. Throughout the material, the repetitions were used over a longer sequence when Martin and his partner are communicating. There were also examples were a repetition of a single sign was performed in a series right after each other. These repetitions of signs and/or gestures were varied in small or bigger movements and the frequency of repetitions variated from just two times to seventeen times. Repetitions of rhythms and signs in the interaction and communication with Martin was the second most common category in the data (see chart 1). This indicates an importance of frequent 
repetitions to facilitate the best conditions to perceive and share something through a bodily/tactile modality.

Rhythm. This theme includes the partner's use of rhythms in the bodily/tactile interaction and communication with Martin. Rhythms in different ways were used together with Martin in a playful frame when the hand-over-hand position was not established. Rhythmic movements and gestures were then used on Martin's body or on the floor next to him accompanied with singing vocally. There were also variations in the clapping regarding speed but also in the manner of how the partner claps for example using the whole hand or just one finger.

Rhythm is known to be an important ingredients of developing language in general and explains the often use of lullabies with children or the rhythmic patterns in sign language (Jantunen, Mesch, Pupponen,\& Laaksonen, 2016).

\section{Discussion}

This case study has shown that the partner and Martin use different conversational positions with their bodies and hands and that the partner transform both the general structure of visual signs (articulations and articulation place) and communicative forms for turn taking, tempo, repetitions and rhythms. It also showed a transformation in the modalities regarding the awareness of other people around you and the possibility to be in a multi-party conversation.

The different film clips of Martin and his partners during the years made it possible to get a glimpse of how his partners are contributing to their bodily/tactile language. They are transforming their customs from using visual sign language to a bodily/tactile modality and by that using different strategies to facilitate Martin's development.

This study indicates an importance of using the whole body in interaction and communication with all people with $\mathrm{cdb}$. Some of the differences in the accommodations that the partners made in this study was made considering Martin's age over the years. This concludes that the accommodations were linked to his physical abilities and the development of those. This parameter is important to highlight because it means that the accommodations and the transformations that the partners are able to make must be made on the basis of the person with cdb's preconditions and preferences of being in contact physically. It is also indicating that it is important for all people who come in contact regularly with people with $\mathrm{cdb}$, to receive guidance to be able to contribute with sufficient support for the person with $\mathrm{cdb}$. The use of different bodily/tactile strategies, and the awareness of those over time, to match the development of the person with $\mathrm{cdb}$, is not something that develops naturally without guidance, since the partner is used to a hearing/seeing perspective. 
This study shows one example of partners contribution during the years and the communicative skills that they master together. It does not mean is must look like this for all people with cdb and their partners. Particularly, it does mean that it is always the partner's responsibility to move closer to the perceptual world of the deafblind person and that a person with cdb requires linguistic input in a bodily/tactile modality from their social environment. However, it is also important to underline that the focus of this study was of one side of the togetherness and for development it is not enough only to focus on the linguistic input from the partner. One must also see the expressions from the person with cdb as a ground for further communication and shared meaning.

\section{Limitations of the study}

Firstly, there are restrictions of the findings due to the limited data. For a more accurate result the study would have to be based on several video recordings. This means that the findings presented in the result section, for example articulations, will only be a small part of Martin's and the partners whole communicative repertoire. In other words the study only reveals what is visible in the ten film clips. Secondly, the video recordings have been interpreted and transcribed by the author, which means that the communication between Martin and his partners have been described by someone who was not a part of the communicative situations. 


\section{References}

Ask, Larsen, F. (2003). The washing-smooth hole-fish and other findings of semantic potential and negotiation strategies in conversation with congenitally deafblind children. (M.A thesis). Center for Semiotics: University of Aarhus.

Ask, Larsen, F. (2015). Congenital deafblindness and bodily-tactile language acquisition. (Doctoral thesis). Department of Psychology: University of Copenhagen.

Brede, K.S. (2008). Let me join your attention. A Sign Language Perspective on the Communicative

Togetherness with a Child who is Congenitally Deafblind. (M.A. thesis) Educational Sciences, Faculty of behavioral and Social sciences: University of Groningen. Netherlands.

Dammeyer, J., Nielsen, A., Strøm, E., Hendar, O., \& Eiríksdóttir, V. K. (2015). A Case Study of Tactile Language and its Possible Structure: A Tentative Outline to Study Tactile Language Systems among Children with Congenital Deafblindness.Communication Disorders, Deaf Studies \& Hearing Aids, vol, 3(2).

Forsgren, G, Daelman, M., Hart, P. (2018). Sign Construction Based on Heightened Tactile Perception by Persons with Congenital Deafblindness. Journal of Deaflblind studies on Communication. Vol. 4, 2018, pp. 4-23

Gregersen, A (2018). Body with Body: Interaction with children in the human niche. Journal of Deafblind studies on Communication. Vol. 4, 2018, pp. 67-83

Guest, G., Macqueen M.K., \& Namey, E.E. (2012). Applied thematic analysis. Sage: London.

Hanning-Zwanenburg, A.H., Rodbroe, I.B., Nafstad, A.V., \& Souriau, J. (2016). Narrative-based Conversations with Children who are Congenitally Deaf-Blind. Journal of deaf blind studies of communication. vol 1. no 1.

Hart, P. (2006). Using imitation with congenitally deafblind adults: Establishing meaningful communication partnerships. Infant and Child Development, Vol,15(3). 263-274.

Hart, P. (2010). Moving beyond the common touchpoint. Discovering language with congenitally deafblind people. (Doctoral study). Dundee: University of Dundee.

Hart, P., \& Rodbroe, I. (2010). Dialogicality in culture. Response from the perspective of congenital deafblindness. Paper from the conference The Magic of Dialogue, Suresnes, Paris, June 22nd 2010.

Hill, S., \& Millar, N. (2015). Case Study Research: The Child in Context. In: Saracho, O, N (Ed.). Handbook of research methods in early childhood education. Information age publishing. Vol. 1. 
Jantunen, T., Mesch, J., Puupponen, A., \& Laaksonen, J. (2016). On the rhythm of head movements in Finnish and Swedish Sign Language sentences. Conference paper. The Proceedings of Speech Prosody. 31 May - 3 Jun 2016, Boston, USA

Lane, L.L., Pillard, R., \& Hedberg, U. (2010). The people of the eye. Deaf ethnicity and ancestry. Oxford University Press: New York.

Lexicon at Stockholm's university homepage (http://teckensprakslexikon.su.se).

Lindström, C. (2017). Contributing to a bodily/tactile language by transforming cultural customs. A case study of Partners Communicative Accommodations in Socialised Praxises based on a Bodily/Tactile Modality and its Influences on a Bodily/Tactile Culture. (M.A. thesis) Educational Sciences, Faculty of behavioral and Social sciences: University of Groningen. Netherlands.

Lundqvist, E. K., Klefstad, L., \& Seljeseth, T. (2012). Feel my Language. University Hospital of North Norway/Regional Centre for People with Deafblindness.

Miles, B. (2003). Talking the Language of the Hands to the Hands. DB-link. Voice: (800) 4389376.

Mesch, J. (1998). Teckenspråk i taktil form : turtagning och frågor i dövblindas samtal på teckenspråk. (Doctoral study). Stockholms universitet.

Mesch, J. (2011). Variations in tactile signing - the case of one-handed signing. Journal of Estonian and Finno-Ugrian Linguistics. 2011, 2 - 1: 273 - 282.

Nafstad, V.A., \& Daelman, M. (2017). Excursions into the Richness of Human Communication: Theory and practice during and before the 10 years of the International Master program on Communication and Congenital deafblindness. Journal of deafblind studies on communication. Vol 3. Nr 1.

Nafstad, V. A., \& Rodbroe, B. I. (2013). Communicative relations. Interventions that create communication with persons with congenital deafblindness. Materialcentret: Aalborg.

Nordic welfare center (2017). Nordic definition of deafblindness. <http://www.nordicwelfare.org/PageFiles/992/nordic-definition-ofdeafblindness.pdf>

Norrby, C. (2014). Samtalsanalys. Så gör vi när vi pratar med varandra. Studentlitteratur: Lund.

Ochs, E., \& Schieffelin, B.B. (1983). Acquiring conversational competence. Routledge \& Kegan Paul: London. 
Ochs, E., \& Schieffelin, B.B. (1984). Language acquisition and socialisation: Three developmental stories. In A.R. Shweder, \& R.A LeVine. (Eds.) Culture theory: Essays on mind, self, and emotion. 276-320. Cambridge, UK: Cambridge Univ. Press.

Ochs, E. (1988). Culture and Language Development: Language Acquisition and Language socialization in a Samoan Village. Cambridge: Cambridge University Press

Ochs, E., Solomon, O., \& Sterponi, L. (2005). Limitations and transformations of habitus in Child-Directed Communication. Discourse studies, Vol, 7(4-5): 547-583.

Ochs, E., \& Schieffelin, B. (2012) The theory of language socialization. In A. Duranti, E. Ochs, \& B. Schieffelin. (Eds.). The handbook of language socialization. Wiley-Blackwell publication.

Raanes, E. (2006) Å gripe inntrykk og uttrykk. Interaksjon og meningsdanning i døvblindes samtaler. (Doctoral thesis). Trondheim, Norway: NTNU The Norwegian Technical University, Department of Language and Communication

Rodbroe, I., \& Janssen, M. (2008). Communication and deafblindness. Congenital and the core principles for interventions. The Danish resource center on congenital deafblindness: Denmark, and Viataal: The Netherlands.

Ryan, G.W., \& Bernard, H.R. (2003). Techniques to Identify Themes. Field Methods, Vol, 15 (1), 85-109.

Schjöll Brede, K \& Souriau, J. (2016). Let Me Join Your Tactile Attention: A Sign Language Perspective on the Communicative Togetherness with a Child who is Congenitally Deafblind.Journal of deafblind studies on communication. vol 2. no 1.

Souriau, J., Rodbroe, I., \& Janssen, M. (2010). Communication and congenital deafblindness. Transition to the cultural language. The Danish resource center on congenital deafblindness: Denmark, and Viataal: The Netherlands.

Stokoe, W. (2005). Sign language structure: An outline of the visual communication system of the American deaf. Journal of Deaf Studies and Deaf Education. vol. 10 no. 1 Oxford University Press.

Caroline Lindström, MSc, Special Educator, Section of Support, Mo Gård, Norralundsvägen 13, Finspång, Sweden; e-mail: <lindstrom.caroline@gmail.com>. 